\title{
Significantly dysregulated genes in osteoarthritic labrum cells identified through gene expression profiling
}

\author{
SHUAI WANG ${ }^{1 *}$, CHUNYAN JIANG ${ }^{2 *}$ and KEFENG ZHANG ${ }^{1}$ \\ ${ }^{1}$ Department of Spinal Surgery, Jining No. 1 People's Hospital, Jining, Shandong 272011; \\ ${ }^{2}$ Emergency Department, Affiliated Hospital of Jining Medical College, Jining, Shandong 272000, P.R. China
}

Received October 3, 2018; Accepted April 25, 2019

DOI: $10.3892 / \mathrm{mmr} .2019 .10389$

\begin{abstract}
The aim of the present study was to explore the molecular basis and identify significant genetic alterations in acetabular labrum cells associated with osteoarthritis (OA). Gene expression data of osteoarthritic and normal human labrum cells were downloaded from a public database and reanalyzed. Significant differentially expressed genes (DEGs) were acquired by performing a thorough analysis of microarray data between the OA acetabular labrum cells and control cells. Key genes in OA labrum cells were revealed by a combination of weighted gene co-expression network analysis (WGCNA) and protein-protein interaction (PPI) analysis. Literature mining and drug screening were further performed for these key genes. In total, 141 DEGs between OA and normal labrum cells were identified. In addition, WGCNA and PPI analysis identified 23 DEGs as key genes in the OA labrum. All the key genes were significantly downregulated in OA labrum cells and were grouped into two different WGCNA-PPI common subnetworks. Kinase insert domain receptor $(K D R), C D 34$, cadherin 5 (CDH5), Fms related tyrosine kinase 1 ( $F L T 1$ ) and asporin were hub nodes in the PPI network of DEGs. These key genes were significantly enriched in functional clusters of transforming growth factor, alkaline phosphatase, bone morphogenic protein and extracellular matrix. Drug screening analysis identified several drugs targeting the key genes, including arachidonic acid, yohimbic acid and mimosine. The results of the present study indicate that the changes of FLT1, KDR, CD34 and CDH5 in acetabular labrum cells may be involved in the pathogenesis of OA and could serve as biomarkers and therapeutic targets of OA. Additionally,
\end{abstract}

Correspondence to: Mr. Kefeng Zhang, Department of Spinal Surgery, Jining No. 1 People's Hospital, 6 Jiankang Road, Renchen, Jining, Shandong 272011, P.R. China

E-mail: nuoyan33zhanjion@163.com

*Contributed equally

Key words: osteoarthritis, acetabular labrum cell, biomarker, co-expression analysis, bioinformatics arachidonic acid, yohimbic acid and mimosine may act as potential drugs for OA.

\section{Introduction}

Osteoarthritis (OA) is one of the most common types of arthritis worldwide and affects $\sim 15 \%$ of the population (1). General symptoms of OA include pain and stiffness in joints and physical disability (2). Although loss of articular cartilage is considered to be the primary cause of OA, other joint tissues including subchondral bone, synovium and acetabular labrum are also involved in the development and progression of OA (2). Moreover, increasing evidence supports a role for genetic factors in OA (1), which may provide clues for the diagnosis and treatment of OA.

A series of genes have been identified to be associated with OA, such as growth differentiation factor $5(3)$, DOI2 $(4,5)$ and SMAD family member 3 (6). Previously, bioinformatics methods have been increasingly used and have facilitated the identification of genetic variations in OA (7). Inflammatory genes such as $C D 55$, prostaglandin E synthase (PTGES) and TNF- $\alpha$ induced protein 6 (TNFAIP6) have been shown to be significantly upregulated in articular cartilage in a genome-wide study, indicating that inflammation may be involved in OA progression (7). Dysregulation of genes related to anti-oxidative defense mechanism has also been identified in OA. Another study using expression profiles from OA and normal articular cartilage showed that superoxide dismutase 2 (SOD2), SOD3, and glutathione peroxidase 3 were significantly downregulated in OA articular cartilage, suggesting a close correlation between the dysregulation of anti-oxidative defense and cartilage matrix damage (8).

Extensive studies have been performed to investigate the molecular mechanisms of OA development and progression (9-12). Studies have reported that chitinases, including chitinase 3 like 1 and chitotriosidase, are upregulated while lubricin is downregulated in OA cartilage and they are considered to be potential markers to stage the severity of OA $(9,11)$. In addition, lubricin, as well as collagen type I, collagen type II are found to be associated with the formation of hyaline cartilage (10). Giunta et al (12) revealed that the expression of pituitary adenylate cyclase-activating polypeptide is reduced in OA and contributes to the inhibition of chondrocyte apoptosis induced by interleukin $1 \beta$. However, much less is known about the transcriptional alterations 
occurring in the acetabular labrum. Two recent studies compared the expression profiles of OA acetabular labrum cells and healthy cells $(13,14)$. One of the studies collected and deposited the expression data in the Gene Expression Omnibus database under accession number GSE60762 (14). The study focused on small leucine rich repeat proteins (SLRPs) and indicated that genes coding for osteomodulin, osteoglycin, and asporin (ASPN) may be functionally important for OA (14). Another study using the same dataset revealed several distinct $\mathrm{OA}$ related genes, including cadherin 2 ( $C D H 2)$, Wnt family member 5A, kinase insert domain receptor (KDR, also known as $V E G F R 2)$, Fms related tyrosine kinase 1 (FLT1) and CDH5 (13). However, further studies are needed to identify additional essential genes related to OA acetabular labrum.

To get new insights into the molecular mechanisms underlying the pathological changes in the OA acetabular labrum, the GSE60762 dataset was reanalyzed using an optimized bioinformatics strategy as diverse bioinformatics approaches may lead to novel insights (15). Following the screening of important differentially expressed genes (DEGs), key genes associated with OA were identified using a combination of co-expression analysis and protein-protein interaction (PPI) analysis. Moreover, the functions of these key genes as well as potential drugs targeting the proteins encoded by these genes were investigated. Therefore, the present study may advance the understanding of the underlying molecular mechanisms of $\mathrm{OA}$ and may contribute to the diagnosis and treatment of OA.

\section{Materials and methods}

Data source and preprocessing. Expression profile data under the accession number GSE60762 was downloaded from the Gene Expression Omnibus (https://www.ncbi. nlm.nih.gov/geo/) (16) database and used for the present study. In total, 5 OA samples and 3 normal samples from acetabular labrum cells were included in the present study. The Series Matrix File(s) was downloaded and the probe names were converted to gene symbols according to the annotation information provided on the GPL6244 platform. The expression values of genes were acquired by averaging the values of their corresponding probes using the aggregate function in base R (version 3.3.3; https://cran.r-project.org/). The expression value of probes with missing value was adjusted using the K-nearest neighbor method (17) (nearest neighbor average with the $\mathrm{k}$ value set as 10) from the impute package (18) in R. The expression profile data was normalized by quantile normalization using the preprocessCore package (19) in R.

Screening of significant DEGs. The significant DEGs obtained were screened using the limma package (20) in R. The differences of mean expression values between OA and normal samples were compared using a t test from the package. P-value was adjusted by the Benjamini-Hochberg method. Genes with $\mid \log _{2} \mathrm{FC}$ (fold change) $\mid>1$ and $\mathrm{P}<0.05$ were considered to be significant DEGs.

Weighted gene co-expression network analysis (WGCNA) of the significant DEGs. All the significant DEGs were analyzed using WGCNA (https://labs.genetics.ucla.
edu/horvath/CoexpressionNetwork/Rpackages/WGCNA/) (21) package in $\mathrm{R}$ to reveal their co-expression relationships. The DEGs were divided into different WGCNA modules. The correlations between different WGCNA modules and OA were assessed using one-way analysis of variance. $\mathrm{P}<0.05$ was set as the cut-off criteria.

Functional analysis of gene modules. Gene Ontology (GO) (22) enrichment analysis were performed for different WGCNA modules using clusterprofiler package (23) in R, with $\mathrm{P}<0.05$ set as the cut-off criteria.

Construction of PPI network. The interactions among proteins encoded by all the DEGs were predicted using the STRING database (24). The PPI network was constructed and visualized with Cytoscape (version 3.2.0; http://www.cytoscape.org) software (25). Furthermore, WGCNA-PPI common subnetworks were constructed. The resulting common subnetworks contained genes with both the weighted correlations as well as PPIs.

Literature mining analysis of key genes. Gene Cluster with Literature Profiles (GenCLiP; version 2.0) is a software for clustering genes under functional keywords based on literature mining (26). The functions of DEGs were analyzed using the GenCLiP (version 2.0, http://ci.smu.edu.cn/GenCLiP2/analysis.php) (26) module 'Gene Cluster With Literature Profiles'. Previously studied functions of genes could therefore be obtained. Functional keywords with $\mathrm{P}<1 \times 10^{-4}$ and those at least for 2 genes were selected.

Drug screening. Connectivity Map (CMAP; https://portals. broadinstitute.org/cmap) is a database used to connect gene expression signatures with small molecules (27). Potential small drug molecules for arthritis treatment were screened using CMAP. The significant DEGs were converted to probes under HG-U133A platform by format conversion. Subsequently, the OA-related small drug molecules were identified by comparing the expression profiles of the DEGs with the gene expression profiles generated by small molecules from the CMAP database. $\mathrm{P}<0.05$ was set as the cut-off criteria.

\section{Results}

DEGs between OA and normal labrum cells. expression data for a total of 19,952 genes were obtained after data preprocessing. Among these genes, 141 (50 upregulated and 93 downregulated genes) were identified to be differentially expressed in OA samples compared with normal samples (Fig. 1A and B). Hierarchical cluster analysis based on the expression value of DEGs could clearly classify OA and normal samples into two different clusters (Fig. 1C).

Co-expression modules of DEGs. In order to identify OA-related co-expression patterns, DEGs were analyzed using WGCNA, an R package for weighted gene co-expression network analysis (21). In total, 5 significant WGCNA modules were acquired and designated as brown, yellow, blue, turquoise, and grey. Most of the modules contained 

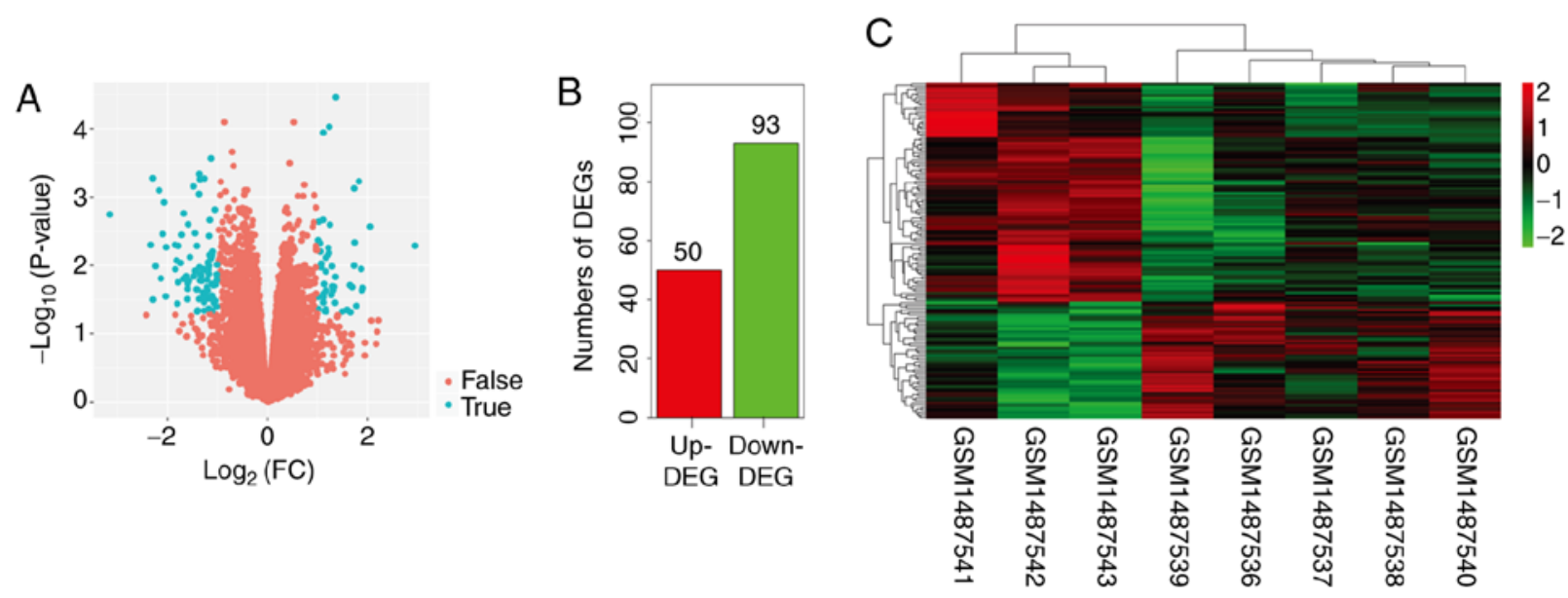

Figure 1. Screening of DEGs between OA and healthy labrum cells. (A) Volcano plot of DEGs. Cyan dots indicate significant DEGs (P<0.05, IlogFCl $>1.0)$ in OA in comparison to healthy labrum cells. (B) The numbers of DEGs are shown in the histogram. Upregulated and downregulated DEGs in OA labrum cells are shown as red and green, respectively. (C) Heatmap of DEGs based on hierarchical clustering analysis. The horizontal axis represents the sample codes and vertical axis, the DEGs. Upregulated and downregulated DEGs in OA labrum cells are shown in red and green, respectively. DEG, differentially expressed genes; OA, osteoarthritis.

$>20$ genes except the grey module, which contained only 8 genes and therefore was not included in the subsequent analysis. The expression of genes in the blue, yellow and turquoise modules correlated negatively with OA, whereas that of genes in the other two modules correlated positively with OA (Table I). Consistent with this, the expression of genes in the blue, yellow and turquoise modules was lower and the expression of genes in the brown module was increased in OA samples compared with the normal samples (Fig. 2A).

Furthermore, GO enrichment analysis was performed for genes in each WGCNA module. The blue module was significantly enriched in multicellular organismal development and system development $(\mathrm{P}<0.05$; Fig. $2 \mathrm{~B})$. The brown module was significantly enriched in terms such as regulation of biological quality, single-organism localization and single-organism transport $(\mathrm{P}<0.01$; Fig. $2 \mathrm{C})$. The turquoise module was significantly enriched in terms related to extracellular region, extracellular region part, development process, single-organism developmental process, multicellular organismal process and single-multicellular organism process $(\mathrm{P}<0.001$; Fig. 2D). The yellow module was significantly enriched in terms such as membrane, membrane part, intrinsic component of membrane and integral components of membrane $(\mathrm{P}<0.001$; Fig. 2E).

PPI network of DEGs. The PPIs between proteins encoded by DEGs were analyzed using the STRING database. In total, 102 PPIs were obtained for 66 DEGs (Fig. 3A). KDR (degree $=12$, downregulated), CD34 (degree=10, downregulated), CDH5 (degree=10, downregulated), FLT1 (degree=10, downregulated) and $A S P N$ (degree=7, downregulated) were considered to be hub nodes in the network, as they had the highest connectivity degree.

The PPIs between proteins encoded by genes in each WGCNA module could also be identified based on the PPI network. Therefore, 4 WGCNA-PPI common subnetworks were constructed to delineate the PPIs within each WGCNA module (Fig. 3B). Both the blue and brown subnetwork consisted of only 4 nodes and 2 PPIs, whereas the yellow and turquoise subnetwork consisted of several nodes and PPIs. In total, 9 nodes and 14 PPIs were included in the yellow common subnetwork, and 12 nodes and 16 PPIs were included in the turquoise common subnetwork. All the downregulated DEGs in the yellow and turquoise subnetwork are shown in OA (Fig. 3B; Table II). Moreover, all the 5 hub nodes were present in either the yellow or turquoise subnetworks and the blue and brown subnetworks did not contain any hub nodes. Specifically, $K D R, C D 34$ and $C D H 5$ were present in the yellow subnetwork, and FLT1 and $A S P N$ were present in the turquoise subnetwork. Consequently, the 23 downregulated genes in yellow and turquoise subnetworks were considered to be key genes associated with OA.

Literature mining analysis of key genes. In order to study the roles of the 23 key genes in the development and progression of OA, literature mining analysis was performed. Using GenCLiP, abundant functional study results were found for all the 23 genes in yellow and turquoise subnetwork (Fig. 4A). FLT1, KDR, CD34 and CDH5 were the key genes associated with the most abundant biological functions. The published literature and related function of the FLT1, KDR, CD34, and CDH5 in OA are shown in Table SI. Functional clusters with the highest scores included transforming growth factor (TGF), alkaline phosphatase, bone morphogenetic protein (BMP) and extracellular matrix (ECM).

Small drug molecules targeting proteins encoded by key genes. Potential small drug molecules targeting proteins encoded by the key genes were also explored using CMAP. As a result, 25 drug molecules were obtained associated with OA, including 15 positively associated and 10 negatively associated drug molecules (Fig. 4B; Table III). Among the top 10 associated drug molecules, 3 were positively associated and 7 were negatively associated with OA (Fig. 4B). The top 3 drugs were arachidonic acid, yohimbic acid and mimosine. 
Table I. WGCNA modules.

\begin{tabular}{lccccc}
\hline Variable & Brown & Yellow & Blue & Turquoise & Grey \\
\hline Correlation $^{\mathrm{a}}$ & 0.74 & -0.8 & -0.87 & -0.79 & 0.82 \\
P-value & 0.035237748 & 0.016851893 & 0.005226361 & 0.019352318 & 0.013654178 \\
Gene number $^{\mathrm{b}}$ & 24 & 21 & 37 & 53 & 8 \\
\hline
\end{tabular}

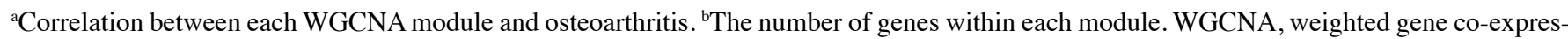
sion network analysis.
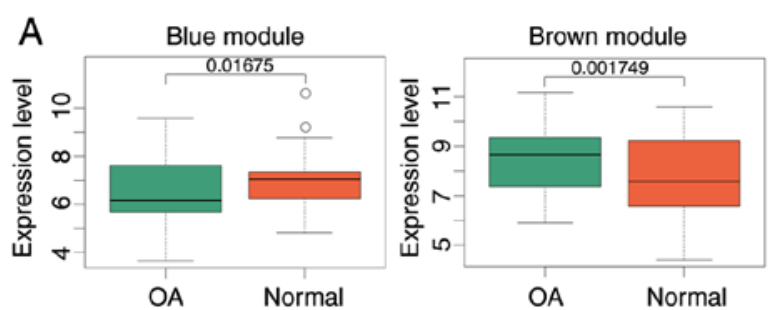

\section{B}

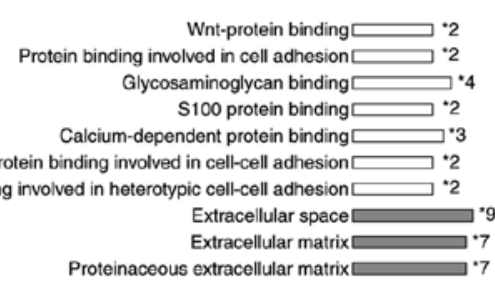

Non-canonical Wnt signaling pathway via MAPK cascade $\square \cdot 2$

Non-canonical Wnt signaling pathway via JNK cascade

Multicellular organismal development Biological adhesion $" 11$ Cell adhesion $" 11$ Neurogenesis $\square$ "12 Generation of neurons $\square$-12 System development 20 Nervous system development $" 16$ \begin{tabular}{l|cccc}
\hline 0 & 1 & 10 & 100 & 1000
\end{tabular}

$\square \mathrm{BP}$

- $\mathrm{CC}$

$\square \mathrm{MF}$

MF

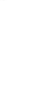
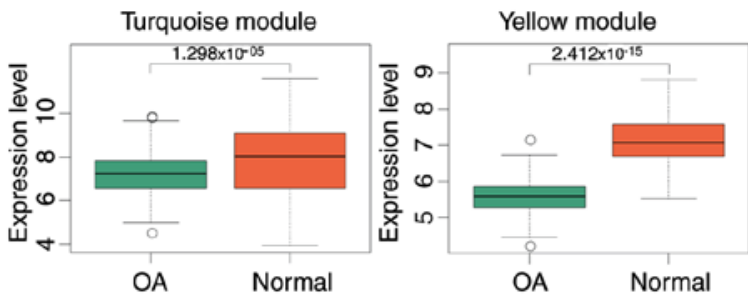

C

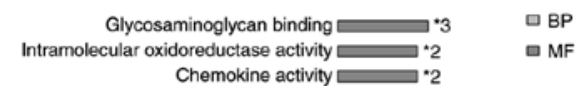
Chemokine activity $\longleftarrow 2$ Receptor regulator activity $\square \cdot 2$ CCR chemokine receptor binding $\square * 2$ Heparin binding $: 3$ Fibronectin binding Sulfur compound binding Lipase activator activity $\square$ ?

Phospholipase activator activity $\square \cdot 2$ Modification by host of symbiont morphology or physiology Regulation of biological quality $* 13$ Single-organism localization $\square * 14$ Single-organism transport Positive regulation of leukocyte migration $\square$ Response to biotic stimulus $\square$ Inflam 7

Response to other organism $\square: 8$
$\square$ onse to external biotic stimulus Leukocyte migration

$$
\begin{array}{lcccc}
\hline & 1 & 10 & 100 & 1000 \\
\text { Gene count }
\end{array}
$$

\section{D}

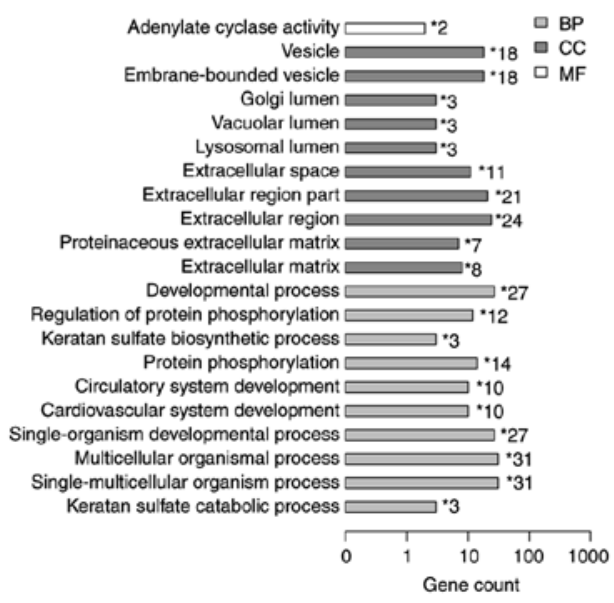

Figure 2. Expression and functional annotation of DEGs in WGCNA modules. (A) Expression of DEGs in OA (green) and normal (red) labrum cells is shown by box-plot. Gene Ontology annotation of DEGs in (B) blue, (C) brown, (D) turquoise, and (E) yellow modules. BP, biological process; CC, cellular component; $\mathrm{MF}$, molecular function; WGCNA, weighted gene co-expression network analysis; DEG, differentially expressed genes.

\section{Discussion}

In the present study, 141 significant DEGs were screened between OA and healthy labrum cells. A total of 23 DEGs were identified to be key genes associated with OA. All the key genes were downregulated in OA labrum cells and could be grouped into two different WGCNA co-expression modules. Moreover, among the key genes, FLT1, KDR, CD34 and CDH5 were associated with the most abundant biological functions and may be substantially involved in the development and progression of OA. 


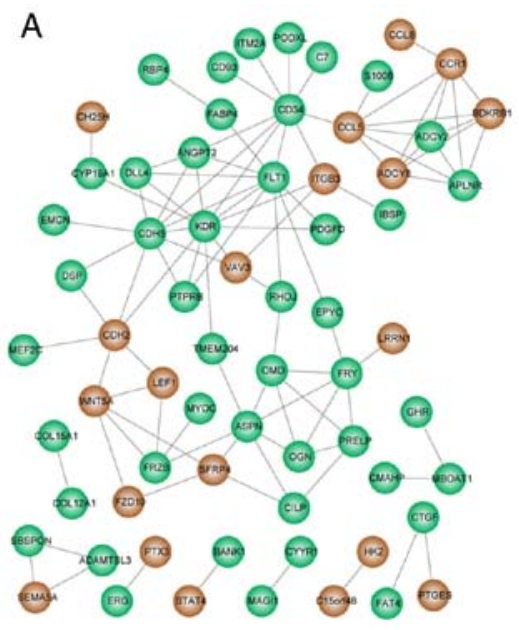

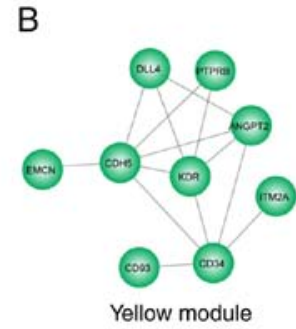

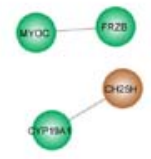

Blue module
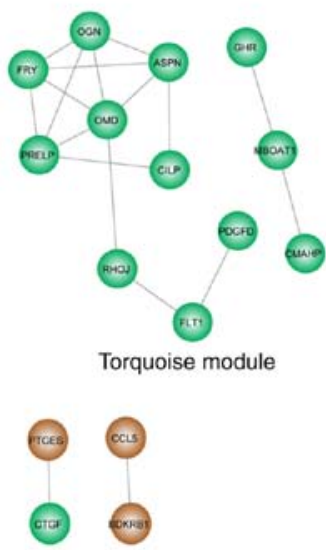

Brown module

Figure 3. PPI analysis of DEGs. (A) PPI network of proteins encoded by DEGs. (B) WGCNA-PPI common subnetworks. Upregulated and downregulated genes are shown as red and green spheres, respectively. Interactions between the proteins are indicated by black lines. DEG, differentially expressed genes; WGCNA, weighted gene co-expression network analysis; PPI, protein-protein interaction.
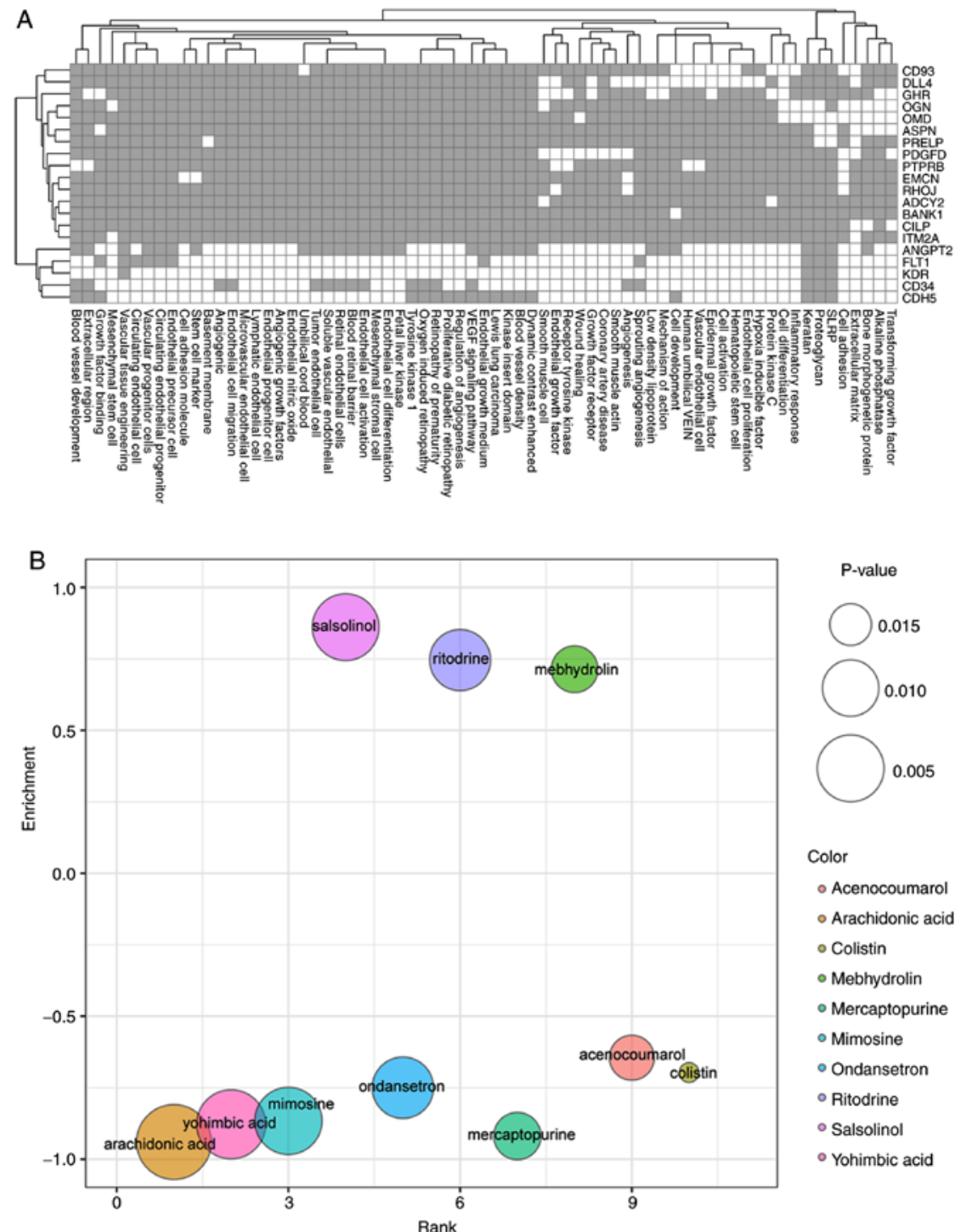

Figure 4. Literature mining and small drug molecule screening of key genes. (A) Functional clustering of key genes based on literature mining. The vertical axis denotes the key genes and horizontal axis shows the functional terms. The white block represents the relationship between a corresponding gene and its functional term, and gray block represents no relationship between a corresponding gene and its functional term. (B) Top 10 small drug molecules targeting the key genes. The horizontal axis shows the ranks of the drug molecules and vertical axis shows the enrichment scores. The bubbles represent drug molecules. The size of a bubble is negatively correlated with its P-value. 
Table II. Genes in the turquoise and yellow WGCNA-PPI common subnetworks.

\begin{tabular}{llll}
\hline Module & Gene symbol & \multicolumn{1}{c}{$\log _{2}$ FC } & \multicolumn{1}{c}{ P-value } \\
\hline Turquoise & ADCY2 & -1.231891879 & 0.02107479 \\
& ASPN & -3.174936812 & 0.001813696 \\
& BANK1 & -1.039158068 & 0.040643831 \\
& CILP & -1.337322006 & 0.017005166 \\
& CMAHP & -1.69454791 & 0.015905385 \\
& FLT1 & -2.083203641 & 0.001212928 \\
& FRY & -1.126732775 & 0.047399489 \\
& GHR & -1.127449211 & 0.007123746 \\
& MBOAT1 & -1.193125199 & 0.030776301 \\
& OGN & -2.05075982 & 0.028726148 \\
& OMD & -2.042591674 & 0.005478374 \\
Yellow & PDGFD & -1.082416359 & 0.035278215 \\
& PRELP & -1.07748275 & 0.018850184 \\
& RHOJ & -1.586566225 & 0.01714161 \\
& ANGPT2 & -1.800427427 & 0.005234895 \\
& CD34 & -1.734034415 & 0.003612027 \\
& CD93 & -1.183506958 & 0.010186758 \\
& CDH5 & -1.428997713 & 0.005249021 \\
& DLL4 & -1.032847908 & 0.016503115 \\
EMCN & -2.351818405 & 0.005082473 \\
& ITM2A & -1.377567348 & 0.010768885 \\
KDR & -2.312597602 & 0.000534604 \\
& PTPRB & -1.028136925 & 0.009858035 \\
\hline
\end{tabular}

FC, fold change; WGCNA, weighted gene co-expression network analysis; PPI, protein-protein interaction.

In the PPI analysis in the present study, it was demonstrated that FLT1, KDR, CD34 and $C D H 5$ together with $A S P N$, were hub nodes in the PPI network of DEGs. As mentioned above, a previous bioinformatic study using the same GSE60762 dataset also demonstrated that $F L T 1, K D R$ and $C D H 5$ were among the hub nodes (13). FLT1 and KDR are vascular endothelial growth factor (VEGF) receptors activated upon VEGF binding (28). VEGF signaling has been reported to promote the formation of cartilage matrix, indicating that inhibition of VEGF signaling may cause cartilage damage in OA (29). It was demonstrated that both $F L T 1$ and $K D R$ were downregulated in the OA labrum, suggesting that inhibition of VEGF signaling may also lead to labrum damage in OA. In addition, it has reported that $F L T 1 /$ VEGF are associated with the recruitment and differentiation of osteoclast cells in bone-resorbing and bone-forming $(30,31)$. Moreover, Hopwood et al (32) reveals that the expression of FLT1 is decreased in OA bone and low expression of $F L T 1$ in OA can promote the activity of VEGF in angiogenesis and osteogenesis. Similarly, the phosphorylation of $K D R /$ Flk-1 has been selected in the regulation of angiogenesis induced by VEGF in several studies $(33,34)$. The angiogenesis and inflammation causes and effects each other in the synovium in $\mathrm{OA}$; the inflamed synovium produces VEGF which accelerates angiogenesis, leading to a further inflammatory response (35). Also, Mifune et al (36)
Table III. Small drug molecules screened by CMAP analysis.

\begin{tabular}{clcl}
\hline Rank & \multicolumn{1}{c}{ Drug molecule $^{\mathrm{a}}$} & Mean score $^{\mathrm{b}}$ & P-value \\
\hline 1 & Arachidonic acid & -0.671 & 0.00032 \\
2 & Yohimbic acid & -0.393 & 0.00373 \\
3 & Mimosine & -0.392 & 0.00477 \\
4 & Salsolinol & 0.505 & 0.00505 \\
5 & Ondansetron & -0.332 & 0.00778 \\
6 & Ritodrine & 0.394 & 0.0079 \\
7 & Mercaptopurine & -0.513 & 0.0134 \\
8 & Mebhydrolin & 0.623 & 0.01359 \\
9 & Acenocoumarol & -0.339 & 0.0141 \\
10 & Colistin & -0.421 & 0.01778 \\
11 & Alimemazine & 0.327 & 0.01908 \\
12 & Imatinib & 0.507 & 0.02058 \\
13 & Econazole & 0.515 & 0.02109 \\
14 & Bretylium tosilate & -0.316 & 0.02532 \\
15 & Clebopride & 0.449 & 0.0266 \\
16 & Etifenin & -0.421 & 0.02672 \\
17 & Oxybenzone & 0.511 & 0.02711 \\
18 & Kanamycin & 0.323 & 0.02851 \\
19 & Spaglumic acid & -0.439 & 0.02897 \\
20 & Semustine & 0.469 & 0.03083 \\
21 & Buflomedil & 0.311 & 0.03943 \\
22 & Quercetin & 0.338 & 0.04007 \\
23 & 11-deoxy-16,16- & 0.338 & 0.0401 \\
& dimethylprostaglandin E2 & & \\
24 & Calmidazolium & 0.24 & 0.04036 \\
25 & Digitoxigenin & 0.373 & 0.04752 \\
\hline
\end{tabular}

${ }^{a}$ The names of small drug molecules used in CMAP database; ${ }^{b}$ Mean scores of the connectivity between corresponding drug molecules and the key genes. CMAP, Connectivity Map.

has demonstrated that the upregulation of FLT1 is involved in promoting collagen synthesis in articular cartilage repair. These findings were all consistent with the results of literature mining analysis, suggesting downregulation of those genes might be associated with angiogenesis and osteogenesis in OA by interacting with VEGF. $C D H 5$ is a specific marker for endothelial cells, essential for vascular integrity and endothelial functions (37). It also functions through binding to $K D R$ to suppress VEGF-driven sprouting (38), further confirming the involvement of labrum VEGF signaling in OA. ASPN is a member of the SLRP family, which was found to be downregulated in OA labrum cells by the analysis of the present study and another bioinformatics study (14). In contrast, $A S P N$ was found to be upregulated in OA chondrocytes (39), indicating that distinct pathological processes could exist in OA cartilage and labrum. The other hub node, CD34, is ubiquitously expressed on the surface of hematopoietic cells and represents a marker of hematopoietic cells (40). It has been shown that CD34 facilitates the recruitment of hematopoietic stem cells in the niches of bone marrow (40), which suggests that a loss of function of $C D 34$ may cause damage to bone and result in OA. 
However, the roles of these hub nodes in OA labrum have not been experimentally studied so far. Further studies are needed to clarify the specific roles of FLT1, KDR, CD34, CDH5 and $A S P N$ in the OA labrum.

Various biological functions are dysregulated in OA. TGFs are essential regulators of cell fate control, such as cell proliferation, differentiation and apoptosis (41). It has been demonstrated that the TGF $\beta$ signaling pathway is dysregulated in OA cartilage and bone (42). Reduced TGF $\beta$ signaling may cause a reduction in ECM synthesis of cartilage and therefore lead to increased susceptibility to OA (43). BMPs are growth factors promoting chondrocyte proliferation and ECM synthesis (44). Both BMP-4 and BMP-7 have been demonstrated to have a beneficial effect on $\mathrm{OA}$ by improving cartilage repair (44). Alkaline phosphatase is essential for controlled bone mineralization (45). Dysregulation of alkaline phosphatase activity leads to mineralized bones $(45,46)$. In the present study, it was demonstrated that TGF signaling, ECM, BMP and alkaline phosphatase may also be dysregulated in OA labrum. According to the literature mining analysis of the present study, TGF signaling, ECM, BMP and alkaline phosphatase were the leading moieties associated with the key genes, including but not limited to $F L T 1, K D R, C D 34$, and $C D H 5$. However, the roles of TGF signaling, ECM, BMP and alkaline phosphatase have not been directly studied in OA labrum to date; therefore, further studies are needed to elucidate their roles in OA.

Although OA is traditionally considered as a non-inflammatory disease, increasing evidence supports the involvement of inflammation in OA (47). Previously, synovitis has been recognized as a major feature of OA $(47,48)$. In addition, inflammatory events in other joint tissues, such as cartilage, bone and ligament also contribute to OA $(47,48)$. A variety of inflammatory mediators are released during OA progression. As mentioned above, inflammatory genes including CD55, PTGES and TNFAIP6 are significantly upregulated in articular cartilage (7). In the present study, links were also found between inflammation and OA. Both arachidonic acid and mimosine, two top small molecular drugs that were identified by CMAP analysis to target the OA-associated key genes, are involved in the inflammatory process. L-mimosine is an anti-inflammatory compound that functions by inhibiting chemokines, such as monocyte chemoattractant protein-1 and macrophage inflammatory protein-2 in OA (49). Consistent with this, a negative correlation between mimosine and $\mathrm{OA}$ was identified, indicating the role of inflammation in OA progression. Unlike mimosine, arachidonic acid is known for its pro-inflammatory effect (50). Enzymatic oxidation of arachidonic acid by COX2 produces prostaglandins (50), type of mediators of inflammation, which promote the production of matrix metallopeptidases (51). However, it was demonstrated that arachidonic acid was also negatively correlated with OA, which further complicated the roles of inflammation in OA. Nevertheless, it was hypothesized that both arachidonic acid and mimosine may serve as potential candidate drugs for OA treatment, though the effects of arachidonic acid and mimosine should be evaluated in future experimental research.

The obvious advantage of the present study is that an optimized bioinformatics strategy combining WGCNA analysis and PPI analysis was used to reveal a well-defined and more comprehensive set of key genes associated with OA. However, the present study was also limited by the small sample size included in the original dataset. Due to the particularity of the osteoarthritic labrum cell samples used, appropriate data for data validation and clinical samples for experimental validation was not found. Therefore, studies with a larger number of samples will be used to verify the results of the present in the future. To conclude, $23 \mathrm{OA}$-associated key genes in labrum cells were identified, among which $F L T 1, K D R, C D 34$ and $C D H 5$ may be involved in the development and progression of OA. It was hypothesized that the findings would greatly contribute to the investigation of OA progression; these genes may serve as novel prognostic and diagnostic biomarker candidates and potential therapeutic targets of OA, but further studies with large sample sizes are needed to verify the results of the present study.

\section{Acknowledgements}

Not applicable.

\section{Funding}

No funding was received.

\section{Availability of data and materials}

All data generated or analyzed during the present study are included in this published article.

\section{Authors' contributions}

SW and CJ made substantial contributions to conception and design, acquisition of data, analysis and interpretation of data and drafted the manuscript. KZ revised the manuscript for important intellectual content and conducted the statistical analysis. All authors read and approved the final manuscript.

\section{Ethics approval and consent to participate}

Not applicable.

\section{Patient consent for publication}

Not applicable.

\section{Competing interest}

The authors declare that they have no competing interest.

\section{References}

1. Johnson VL and Hunter DJ: The epidemiology of osteoarthritis. Best Pract Res Clin Rheumatol 28: 5-15, 2014.

2. Madry H, Luyten FP and Facchini A: Biological aspects of early osteoarthritis. Knee Surg Sports Traumatol Arthrosc 20: 407-422, 2012

3. Zhang Y, Jia J, Yang S, Liu X, Ye S and Tian H: MicroRNA-21 controls the development of osteoarthritis by targeting GDF-5 in chondrocytes. Exp Mol Med 46: e79, 2014.

4. Meulenbelt I, Min JL, Bos S, Riyazi N, Houwing-Duistermaat JJ, van der Wijk HJ, Kroon HM, Nakajima M, Ikegawa S, Uitterlinden AG, et al: Identification of DIO2 as a new susceptibility locus for symptomatic osteoarthritis. Hum Mol Genet 17: $1867-1875,2008$ 
5. Bomer N, den Hollander W, Ramos YF, Bos SD, van der Breggen R, Lakenberg N, Pepers BA, van Eeden AE, Darvishan A, Tobi EW, et al: Underlying molecular mechanisms of DIO2 susceptibility in symptomatic osteoarthritis. Ann Rheum Dis 74: 1571-1579, 2015.

6. Sharma AC, Srivastava RN, Srivastava SR, Parmar D, Singh A and Raj S: Association between single nucleotide polymorphisms of SMAD3 and BMP5 with the risk of knee osteoarthritis. J Clin Diagn Res 11: GC01-GC04, 2017.

7. Ramos YF, den Hollander W, Bovée JV, Bomer $\mathrm{N}$, van der Breggen R, Lakenberg N, Keurentjes JC, Goeman JJ, Slagboom PE, Nelissen RG, et al: Genes involved in the osteoarthritis process identified through genome wide expression analysis in articular cartilage; the RAAK study. PLoS One 9: e103056, 2014

8. Aigner T, Fundel K, Saas J, Gebhard PM, Haag J, Weiss T, Zien A, Obermayr F, Zimmer R and Bartnik E: Large-scale gene expression profiling reveals major pathogenetic pathways of cartilage degeneration in osteoarthritis. Arthritis Rheum 54: 3533-3544, 2006.

9. Szychlinska MA,TrovatoFM,DiRosa M, Malaguarnera L,Puzzo L, Leonardi R, Castrogiovanni P and Musumeci G: Co-expression and co-localization of cartilage glycoproteins CHI3L1 and lubricin in osteoarthritic cartilage: Morphological, immunohistochemical and gene expression profiles. Int J Mol Sci 17: 359, 2016.

10. Musumeci G, Mobasheri A, Trovato FM, Szychlinska MA Graziano AC, Lo Furno D, Avola R, Mangano S, Giuffrida R and Cardile V: Biosynthesis of collagen I, II, RUNX2 and lubricin at different time points of chondrogenic differentiation in a 3D in vitro model of human mesenchymal stem cells derived from adipose tissue. Acta Histochem 116: 1407-1417, 2014.

11. Di Rosa M, Szychlinska MA, Tibullo D, Malaguarnera L and Musumeci G: Expression of CHI3L1 and CHIT1 in osteoarthritic rat cartilage model. A morphological study. Eur J Histochem 58 : 2423, 2014

12. Giunta S, Castorina A, Marzagalli R, Szychlinska MA, Pichler K, Mobasheri A and Musumeci G: Ameliorative effects of PACAP against cartilage degeneration. Morphological, immunohistochemical and biochemical evidence from in vivo and in vitro models of rat osteoarthritis. Int J Mol Sci 16: 5922-5944, 2015.

13. Wang B, Zhao J and Zhang P: Gene signatures in osteoarthritic acetabular labrum using microarray analysis. Int $\mathbf{J}$ Rheum Dis 20: 1927-1934, 2017.

14. Juchtmans N, Dhollander AA, Coudenys J, Audenaert EA, Pattyn C, Lambrecht S and Elewaut D: Distinct dysregulation of the small leucine-rich repeat protein family in osteoarthritic acetabular labrum compared to articular cartilage. Arthritis Rheumatol 67: 435-441, 2015.

15. Najafi A, Tavallaei M and Hosseini SM: A systems biology approach for miRNA-mRNA expression patterns analysis in non-small cell lung cancer. Cancer Biomark 16: 31-45, 2016.

16. Barrett T and Edgar R: Gene expression omnibus: Microarray data storage, submission, retrieval, and analysis. Methods Enzymol 411: 352-369, 2006

17. Altman NS: An introduction to kernel and nearest-neighbor nonparametric regression. Am Statistician 46: 175-185, 1992.

18. Hastie T, Tibshirani R, Balasubramanian N and Chu G: Impute: Imputation for microarray data. $\mathrm{R}$ package version 1.42. 0. 2013 .

19. Bolstad BM: preprocess Core: A collection of pre-processing functions. R package version 1, 2013.

20. Smyth GK, Ritchie M, Thorne N and Wettenhall J: LIMMA: Linear models for microarray data. In bioinformatics and computational biology solutions using R and bioconductor. Statistics for Biology and Health, 2005.

21. Langfelder $P$ and Horvath S: WGCNA: An R package for weighted correlation network analysis. BMC Bioinformatics 9: 559, 2008.

22. Ashburner M, Ball CA, Blake JA, Botstein D, Butler H, Cherry JM, Davis AP, Dolinski K, Dwight SS, Eppig JT, et al: Gene Ontology: Tool for the unification of biology. The Gene Ontology Consortium. Nat Genet 25: 25-29, 2000.

23. Yu G, Wang LG, Han Y and He QY: clusterProfiler: An R package for comparing biological themes among gene clusters. OMICS 16: 284-287, 2012.

24. von Mering C, Jensen LJ, Kuhn M, Chaffron S, Doerks T, Krüger B, Snel B and Bork P: STRING 7-recent developments in the integration and prediction of protein interactions. Nucleic Acids Res 35 (Database Isuue): D358-D362, 2007.

25. Shannon P, Markiel A, Ozier O, Baliga NS, Wang JT, Ramage D, Amin N, Schwikowski B and Ideker T: Cytoscape: A software environment for integrated models of biomolecular interaction networks. Genome Res 13: 2498-2504, 2003.
26. Wang JH, Zhao LF, Lin P, Su XR, Chen SJ, Huang LQ, Wang HF, Zhang H,Hu ZF, Yao KT and Huang ZX: GenCLiP 2.0: A web server for functional clustering of genes and construction of molecular networks based on free terms. Bioinformatics 30: 2534-2536, 2014.

27. Lamb J, Crawford ED, Peck D, Modell JW, Blat IC, Wrobel MJ, Lerner J, Brunet JP, Subramanian A, Ross KN, et al: The Connectivity Map: Using gene-expression signatures to connect small molecules, genes, and disease. Science 313: 1929-1935, 2006

28. Ferrara N, Gerber HP and LeCouter J: The biology of VEGF and its receptors. Nat Med 9: 669-676, 2003.

29. Pesesse L, Sanchez C and Henrotin Y: Osteochondral plate angiogenesis: A new treatment target in osteoarthritis. Joint Bone Spine 78: 144-149, 2011

30. Mayr-Wohlfart U, Waltenberger J, Hausser H, Kessler S, Günther KP, Dehio C, Puhl W and Brenner RE: Vascular endothelial growth factor stimulates chemotactic migration of primary human osteoblasts. Bone 30: 472-477, 2002.

31. Deckers MM, Karperien M, Van der Bent C, Yamashita T, Papapoulos SE and Löwik CW: Expression of vascular endothelial growth factors and their receptors during osteoblast differentiation. Endocrinology 141: 1667-1674, 2000

32. Hopwood B, Gronthos S, Kuliwaba JS, Robey PG, Findlay DM and Fazzalari NL: Identification of differentially expressed genes between osteoarthritic and normal trabecular bone from the intertrochanteric region of the proximal femur using cDNA microarray analysis. Bone 36: 635-644, 2005.

33. Gao Y, Lu N, Ling Y, Chen Y, Wang L, Zhao Q, Qi Q, Liu W, Zhang H, You Q and Guo Q: Oroxylin A inhibits angiogenesis through blocking vascular endothelial growth factor-induced KDR/Flk-1 phosphorylation. J Cancer Res Clin Oncol 136: 667-675, 2010

34. Kwak HJ, Park MJ, Park CM, Moon SI, Yoo DH, Lee HC, Lee SH, Kim MS, Lee HW, Shin WS, et al: Emodin inhibits vascular endothelial growth factor-A-induced angiogenesis by blocking receptor-2 (KDR/Flk-1) phosphorylation. Int J Cancer 118: 2711-2720, 2010 .

35. Haywood L, Mcwilliams DF, Pearson CI, Gill SE, Ganesan A, Wilson D and Walsh DA: Inflammation and angiogenesis in osteoarthritis. Arthritis Rheum 48: 2173-2177, 2003.

36. Mifune Y, Matsumoto T, Takayama K, Ota S, Li H, Meszaros LB, Usas A, Nagamune K, Gharaibeh B, Fu FH and Huard J: The effect of platelet-rich plasma on the regenerative therapy of muscle derived stem cells for articular cartilage repair. Osteoarthritis Cartilage 21: 175-185, 2013

37. Mao XG, Xue XY, Wang L, Zhang X, Yan M, Tu YY, Lin W, Jiang XF, Ren HG, Zhang W and Song SJ: CDH5 is specifically activated in glioblastoma stemlike cells and contributes to vasculogenic mimicry induced by hypoxia. Neuro Oncol 15: 865-879, 2013.

38. Abraham S, Yeo M, Montero-Balaguer M, Paterson H, Dejana E, Marshall CJ and Mavria G: VE-Cadherin-mediated cell-cell interaction suppresses sprouting via signaling to MLC2 phosphorylation. Curr Biol 19: 668-674, 2009.

39. Strassburger M, Bloch W, Sulyok S, Schüller J, Keist AF, Schmidt A, Wenk J, Peters T, Wlaschek M, Lenart J, et al: Heterozygous deficiency of manganese superoxide dismutase results in severe lipid peroxidation and spontaneous apoptosis in murine myocardium in vivo. Free Radic Biol Med 38: 1458-1470, 2005.

40. Sidney LE, Branch MJ, Dunphy SE, Dua HS and Hopkinson A: Concise review: Evidence for CD34 as a common marker for diverse progenitors. Stem cells 32: 1380-1389, 2014.

41. Kamato D, Burch ML, Piva TJ, Rezaei HB, Rostam MA, Xu S, Zheng W, Little PJ and Osman N: Transforming growth factor- $\beta$ signalling: Role and consequences of Smad linker region phosphorylation. Cell Signal 25: 2017-2024, 2013.

42. Grafe I, Yang T, Alexander S, Homan EP, Lietman C, Jiang MM, Bertin T, Munivez E, Chen Y, Dawson B, et al: Excessive transforming growth factor-beta signaling is a common mechanism in osteogenesis imperfecta. Nat Med 20: 670-675, 2014.

43. Blaney Davidson EN, van der Kraan PM and van den Berg WB: TGF-beta and osteoarthritis. Osteoarthritis Cartilage 15 597-604, 2007.

44. Kuroda R, Usas A, Kubo S, Corsi K, Peng H, Rose T, Cummins J, $\mathrm{Fu} \mathrm{FH}$ and Huard J: Cartilage repair using bone morphogenetic protein 4 and muscle-derived stem cells. Arthritis Rheum 54: 433-442, 2006

45. Hessle L, Johnson KA, Anderson HC, Narisawa S, Sali A, Goding JW, Terkeltaub R and Millan JL: Tissue-nonspecific alkaline phosphatase and plasma cell membrane glycoprotein-1 are central antagonistic regulators of bone mineralization. Proc Natl Acad Sci USA 99: 9445-9449, 2002. 
46. Addison WN, Azari F, Sørensen ES, Kaartinen MT and McKee MD: Pyrophosphate inhibits mineralization of osteoblast cultures by binding to mineral, up-regulating osteopontin, and inhibiting alkaline phosphatase activity. J Biol Chem 282: 15872-15883, 2007.

47. Berenbaum F: Osteoarthritis as an inflammatory disease (osteoarthritis is not osteoarthrosis!). Osteoarthritis Cartilage 21: 16-21, 2013.

48. Goldring MB and Otero M: Inflammation in osteoarthritis. Curr Opin Rheumatol 23: 471-478, 2011.

49. Melgarejo E, Medina MA, Sánchez-Jiménez F and Urdiales JL: Monocyte chemoattractant protein-1: A key mediator in inflammatory processes. Int J Biochem Cell Biol 41: 998-1001, 2009.
50. Kuehl FA Jr and Egan RW: Prostaglandins, arachidonic acid, and inflammation. Science 210: 978-984, 1980.

51. Pillinger MH, Marjanovic N, Kim SY, Scher JU, Izmirly P Tolani S, Dinsell V, Lee YC, Blaser MJ and Abramson SB: Matrix metalloproteinase secretion by gastric epithelial cells is regulated by E prostaglandins and MAPKs. J Biol Chem 280: 9973-9979, 2005.

This work is licensed under a Creative Commons Attribution-NonCommercial-NoDerivatives 4.0 International (CC BY-NC-ND 4.0) License. 